\title{
Kernos
}

Revue internationale et pluridisciplinaire de religion grecque antique

3 | 1990

Varia

\section{L'oracle de Dodone. Mythe et rituel}

\section{Ariadni Gartziou-Tatti}

URL : http://journals.openedition.org/kernos/985

DOI : $10.4000 /$ kernos.985

ISSN : 2034-7871

\section{Éditeur}

Centre international d'étude de la religion grecque antique

Édition imprimée

Date de publication : 1 janvier 1990

ISSN : 0776-3824

\section{Référence électronique}

Ariadni Gartziou-Tatti, «L'oracle de Dodone. Mythe et rituel », Kernos [En ligne], 3 | 1990, mis en ligne le 19 avril 2011, consulté le 01 mai 2019. URL : http://journals.openedition.org/kernos/985 ; DOI :

10.4000/kernos.985 


\section{L'ORACLE DE DODONE. MYTHE ET RITUEL*}

Dans la région lointaine d'Épire et dans le site montagneux de Dodone, les fouilles archéologiques ${ }^{1}$ ont confirmé l'existence d'un oracle fameux consacré à Zeus, oracle dont l'importance a déjà été signalée par la tradition littéraire. Dans l'Iliade ${ }^{2}$, Achille adresse une prière à Zeus en tant que dieu de Dodone où habitaient les Selloi, ses interprètes aux pieds jamais lavés qui couchaient par terre ${ }^{3}$. De même dans l'Odyssée 4 , Ulysse adressait au feuillage divin du grand chêne de Zeus la prière de lui accorder un conseil à propos de son retour à Ithaque. Le fragment 240 M.-W. du Catalogue des femmes d'Hésiode contient certains renseignements sur ce même sujet. Bien que les vers 7-9 du

* Je voudrais exprimer ma reconnaissance à Ch. Tzouvara-Souli, professeur à l'Université de Ioannina, dont les suggestions et les remarques sur l'espace des divinités masculines en Épire m'ont été très utiles.

1 Les travaux de S.I. DAKARIS tiennent ici une place privilégiée. Voir en particu-

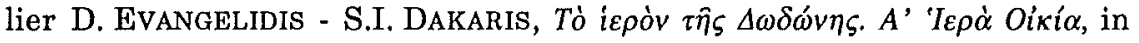
$A E$ (1959) [1964], p. 1-194 et c.r. par M. ANDRONIKOS, in Gnomon, 38 (1966), p. 270-274; S.I. DAKARIS, Tó i $\varepsilon \rho o ̀ v ~ \tau \hat{\eta} \varsigma \Delta \omega \delta \omega ́ v \eta \varsigma$, in $A D, 16$ (1960), p. 4-40; Das Taubenorakel von Dodona und das Totenorakel bei Ephyra, in AK (1963), 1.

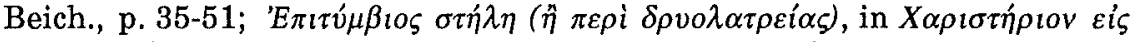

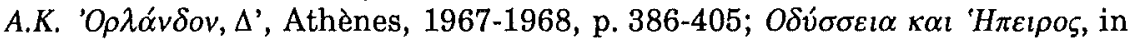

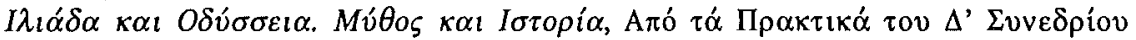

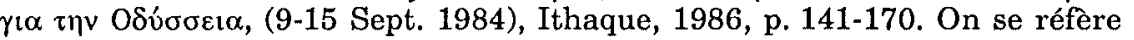
également aux travaux de P.R. Franke, Das Taubenorakel en Dodona und die Eiche als heilige Baum des Zeus Naios, in MDAI(A), 71 (1956), p. 60-65 (= Die antiken Münzen von Epirus, Wiesbaden, 1961, p. 317-322); D.M. NICOL, The Oracle of Dodona, in $G \& R, 5$ (1958), p. 127-143; G. RACHET, Le sanctuaire de Dodone : origine et moyens de divination, in $B A G B$ (1962), p. 86-99; V. Pötscher, Zeus Naios und Dione in Dodona, in Mnemosyne, Ser. IV, 19, 2 (1966), p. 113-147; H.W. PARKe, The Oracles of Zeus. Dodona, Olympia,

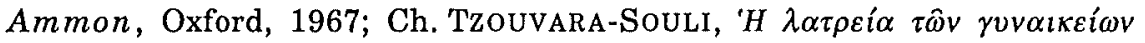

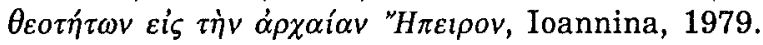

2 Il., XVI, 233-235 et schol. Cf. H. ERBse, Scholia Graeca in Homeri Iliadem, Berlin, 1975, vol. IV, p. 218 sq. Les sources littéraires sont rassemblées et étudiées par J. FRIEDRICH, Dodoniaca, Freiburg, 1932.

3 Cf. aussi SoPH., Trach., 1167; EuR., Érechthée, fr. 355 Nauck $^{2}$; CALLIM., Hymne à Délos, 286.

4 Od., XIV, 327-328; XIX, 296-297. 
texte soient mutilés ${ }^{5}$, on peut conclure qu'il y avait un lien explicite entre le chêne et les interprètes de l'oracle. Hérodote, dans un récit concernant la fondation de cet oracle ${ }^{6}$, insère le mythe des deux colombes noires qui s'étaient envolées de Thèbes en Égypte, l'une en Libye et l'autre chez les Dodonéens. Cette dernière se percha dans un chêne et, d'une voix humaine, y ordonna l'établissement d'un oracle de Zeus. Hérodote tente de mettre ce récit des deux colombes en rapport avec celui de deux femmes, également originaires de Thèbes, qui avaient institué les premiers oracles en Libye et en Grèce. Selon Hérodote, ces deux femmes furent appelées colombes par les Dodonéens parce qu'étant barbares, leur langage était pareil au chant des oiseaux.

On peut donc saisir dans les grandes lignes le fonctionnement de cet oracle dans le sanctuaire de Dodone. Les oracles de Zeus étaient rendus par le bruissement du feuillage du chêne et le cri des bisets ${ }^{7}$ pour être ensuite interprétés par les femmes-colombes, connues sous le nom de

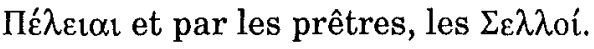

On se fait donc une idée générale de l'évolution de ce culte qui se manifeste tantôt autour d'une puissance féminine, la déesse-mère avec ses symboles (colombe ou arbre sacré) ${ }^{8}$ tantôt autour du culte de l'arbre

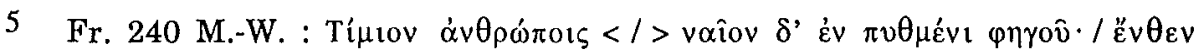

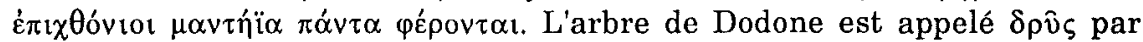
Homère tandis que Hésiode le dénomme pryós. Cf. OLCK, art. Eiche, in RE, V, 2 (1958), col. 2013-2075; RACHET, art. cit., p. 92 et PöTSCHER, art. cit., p. 123 sq. Ce même chêne avait prédit, selon SopHOCLE (Trach., 171, 1166-1168), la durée et la fin des travaux d'Héraclès. Pour les autres héros qui sont allés consulter l'oracle, cf. L. BODSON, 'IEPA ZRIA. Contribution à l'étude de la place de l'animal dans la religion grecque ancienne, Bruxelles, 1978, p. 110, n. 130-138.

6 HDT., II, 54-57. L'historien fut le premier à proposer une interprétation rationnelle des données mythiques de l'oracle en essayant de concilier des récits contradictoires sur la nature et le rôle des femmes-colombes, interprétation déjà inintelligible à son époque. Cf. R. CRAHAY, La littérature oraculaire chez Hérodote, Paris, 1956, p. 94-95. Le débat sur le nombre des colombes et la question de savoir s'il s'agit de femmes ou d'oiseaux restent encore ouverts. Cf. L. BoDSON, op. cit., p. 101 sq.

7 Cf. RACHET, art. cit., p. 92-98. Une divination inspirée était également possible, cf. Plat., Phèdre, 244b. Sur la discussion, cf. TZouvara-Souli, op. cit., n. 484.

8 L'idée selon laquelle la déesse-mère, avec ses symboles, colombe ou arbre sacré, était la puissance primordiale de l'oracle (cf. RACHET, art. cit., p. 88 sq.; DAKARIS, $\Pi_{\varepsilon} \rho \dot{i} \delta \rho v 0 \lambda \alpha \tau \rho \varepsilon i \alpha \varsigma, a r t$. cit., p. 389, n. 14 et p. 395 sq.; TsouvaRA-Soul, op. cit., n. 85, 478-479, 488) s'inscrit dans le cadre d'une interprétation «historique» qui distingue les différentes étapes de l'évolution de l'oracle. - Sur le rôle du chêne dans le cadre de l'oracle, nous suivons la thèse soutenue par A.B. CooK qui insiste sur les rapports entre le chêne et Zeus. Cf. A.B. CooK, Zeus, Jupiter and 
étroitement associé à Zeus, dieu qui aurait d'abord été accompagné de prêtres et ensuite de prêtresses ${ }^{9}$. Selon une deuxième voie d'interprétation, on y voit un mélange de divers éléments religieux provenant du monde méditerranéen ${ }^{10}$ ou les traces de couches successives de l'immigration indo-européenne ${ }^{11}$.

Ces théories qui visent à restituer l'origine du dieu ou de la déesse de l'oracle ne couvrent pas tout le champ sémantique de l'oracle et laissent ouverte la signification des principaux thèmes. Bien que l'Iliade demeure la source littéraire la plus ancienne, complétée par les trouvailles archéologiques qui s'étendent de l'époque mycénienne jusqu'à l'époque gréco-romaine, on ne peut pas préciser exactement les procédés divinatoires, apparemment incohérents, si on ne les examine pas dans le contexte de l'ensemble de l'oracle. On peut donc se faire une image de l'oracle dans le cadre des rites, des croyances et des pratiques religieuses en évitant une voie d'analyse basée sur l'évolution purement historique. En vérité, cette perspective «historique» s'interrogeant sur les premiers maîtres de l'oracle appartient à un mythe qui

the Oak, in $C R, 17$ (1903), p. 174-184, 268-278, 403-421 et 18 (1904), p. 75-89; ID., Zeus. A Study in Ancient Religion, I-III, Cambridge, 1914-1940 (New York, 1964), passim et surtout II, p. 677. Cf. aussi PARKE, op. cit., p. 21 sq. et M.B. Sakellariou, Peuples préhelléniques d'origine indo-européenne, Athènes, 1977, p. 105 sq. - Sur l'association du dieu maître de l'oracle aux bisets qui ont partie liée avec le sanctuaire, cf. A.H. KRAPPE, Les Péleiades, in RA, 36 (1932), p. 77-93; PARKE, op. cit., p. 34 sq. Toutefois, les statuettes féminines portant des colombes, qui ont été trouvées dans le sanctuaire (cf. Tzouvara-Soul, op. cit., p. 56 et n. 398), confirment un certain rapport des colombes avec le monde féminin, mais elles ne signifient en aucun cas une priorité des puissances féminines. À notre avis, le lien du chêne et des colombes avec le monde féminin doit être interprété par rapport à la liaison de ce dernier avec celui de Zeus (infra n. 46), dont le culte était largement répandu à travers le monde grec dans son ensemble depuis l'époque mycénienne, cf. E. ScHWABL, art. Zeus, in RE, Suppl. XV (1976), col. 1001 sq.

9 Cf. BoDson, op. cit., p. 112, n. 155 et p. 116.

10 Cf. PARkE, op. cit., p. 20 sq.

11 D. Evans, Dodon, Dodola et Daedala, in Myth in Indo-European Antiquity, G.I. LARSON (ed.), Berkeley, 1974, p. 99-130; SAKELlariou, op. cit., p. 105 sq., 161 sq., 281 sq., et passim. Même si l'origine indo-européenne du maître de l'oracle ne fait pas de doute, nous croyons que la religion grecque a pris beaucoup de liberté par rapport à cet héritage culturel. Sur ce sujet, cf. B. SERgent, Les trois fonctions des Indo-Européens dans la Grèce ancienne, in Annales(ESC), 6 (1979), p. 1155-1186. 
n'est pas le reflet d'une réalité historique mais qui exprime certaines visions sur l'organisation des oracles ${ }^{12}$.

Compte tenu de ces considérations, notre travail consistera en une analyse de l'ensemble des procédés divinatoires. Tout d'abord, on distingue le rôle central que les oiseaux assument dans le cadre de l'oracle, en occupant l'espace intermédiaire entre le monde céleste et le monde terrestre. Il s'agit du seul genre d'animal qui se trouve en contact direct avec les mondes humain et divin ${ }^{13}$. Témoins de l'audelà, ils exercent une fonction spécifique dans le sanctuaire de Dodone. Une telle perspective d'interprétation permet de préciser la signification de la présence des colombes ainsi que celle de l'aigle qui, à Dodone, s'identifie presque à Zeus ${ }^{14}$. L'aigle symbolise le pouvoir de Zeus dont il incarne la volonté en tant qu'instrument de son épiphanie ${ }^{15}$. À toutes ces fonctions, nous voudrions ajouter celle qui fait de l'aigle une source nourricière, rôle affirmé par le fait que c'est lui qui apporte au petit Zeus le nectar, nourriture sauvage qui garantit l'immortalité ${ }^{16}$. Notons enfin que, dans le monde des dieux, l'aigle intervient non seulement en tant que dispensateur de la vie céleste mais aussi comme facteur qui assure la pérennité de cette vie. Oiseau solaire d'une couleur dorée ${ }^{17}$, ayant des rapports étroits avec le feu céleste dont Zeus est le maître ${ }^{18}$,

12 Cf. Ch. Sourvinou-Inwood, Myth as History: The Previous Owners of the Delphic Oracle, in J. Bremmer (ed.), Interpretations of Greek Mythology, London, 1987, p. 215-241, à propos de Delphes.

13 Pour une vue générale sur le rôle des oiseaux dans le monde grec, cf. D'ARCY W. Thomson, A Glossary of Greek Birds, London, 1936 [1966]; B.C. DiETRICH, The Origins of Greek Religion, Berlin, 1974, p. 118 sq., 235 sq.; P. Pollard, Birds in Greek Life and Myth, Plymouth, 1977; BoDson, op. cit.

14 À Dodone, l'aigle, compagnon de Zeus, est représenté sur les épisèmes des

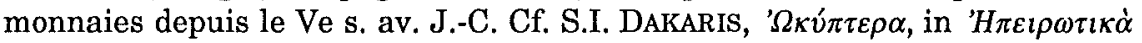
X

15 L'aigle est par excellence l'oiseau symbole et messager de Zeus. Cf. CooK, Zeus, I, p. 84, 91, 532; II, 186, 752, 1133 n. 1 et passim; THOMson, op. cit., s.v. áEтós; G. MYLONAS, The Eagle of Zeus, in CJ, 41 (1945-1946), p. 203-207; POLLARD, op. cit., p. 116 sq., 141 sq.; BoDson, op. cit., p. 96 sq.

16 Sur la place que l'aigle tient dans l'éducation de Zeus, cf. H. VERBRugGen, Le Zeus crétois, Paris, 1981, p. 32 sq., 40 n. 72.

17 L'aigle est par excellence l'oiseau qui reçoit des qualifications chromatiques. Cf. THOMSON, op. cit.; A. SAUVAGE, Études de thèmes animaliers dans la poésie latine. Le cheval, les oiseaux, Bruxelles, 1975 (Coll. Latomus, 143), p. 164 sq.

18 Sur l'aigle oiseau-flamme et porte-flamme, cf. M. Detienne, Les jardins d'Adonis, Paris, 1989, p. 60 sq.; M. DETIENNE - J.-P. Vernant, La cuisine $d u$ sacrifice en pays grec, Paris, 1979 , p. 87 et n. 4, 5. - L'aigle est habituellement 
l'aigle symbolise également la transition du stade de la nourriture sauvage au stade marqué par la présence et l'emploi du feu.

En opposition au caractère lumineux de l'aigle se situe la colombe en tant que représentante de l'univers des oiseaux sauvages ${ }^{19}$ au plumage noir ${ }^{20}$ qui, perchés dans le chêne ${ }^{21}$, établissent également un système de communication entre hommes et dieux. Malgré ces contradicitions, l'univers des colombes réitère la spécificité du rôle de l'aigle. En ce qui concerne la nourriture, la colombe a le privilège de fournir l'ambroisie qui permet d'accéder à l'immortalité 22 . Quant à la transmission de l'oracle, la colombe est le porteur du savoir et de la volonté de Zeus qui assure la communication entre hommes et dieux ${ }^{23}$, complétant ainsi le rôle de l'aigle.

À ce monde d'échanges et de mobilité permanente s'oppose la stabilité du monde terrestre dont les éléments liés à l'oracle sont particulièrement intéressants. Tout d'abord, ce qui retiendra notre attention est l'enracinement solide du chêne qui, grâce à sa nature oraculaire, sa relation étroite avec Zeus et son culte, devient le symbole principal de la communication entre hommes et dieux. Aussi les glands du chêne symbolisent-ils la source permanente de la nourriture sauvage $^{24}$ et son enracinement le rapport étroit entre l'homme et la

associé à la foudre de Zeus sur les épisèmes des monnaies d'Épire. Cf. supra n. 14.

19 Sur les traits de cet oiseau sauvage et son rôle oraculaire, cf. BODSON, op. cit., p. 101 sq.

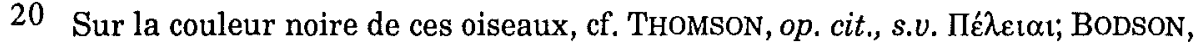
op. cit., p. 101 et n. 70-71.

21 Les colombes, l'aigle et le chêne forment une certaine unité sur les épisèmes des monnaies de bronze de la symmachie épirote. Cf. FRANKE, Die antiken Münzen, op. cit., p. 317 et pl. 15 (V 41-R 51, V 42-R 52); DAKARIS, Das Taubenorakel, art. cit., p. 49 , pl. 22.4 .

22 Sur les colombes, fournisseurs de l'ambroisie à Zeus, cf. Od., XII, 62-63. Sur l'ambroisie et l'immortalité, cf. J. RUDHARDT, Le thème de l'eau primordiale dans la mythologie grecque, Verne, 1971, p. 94 sq.; A. BALlaBriaA, Le Soleil et le Tartare, Paris, 1986, p. 96 sq.

23 Cf. Bodson, op. cit., p. 116, n. 191-192; BALlaBRIGA, op. cit., p. 95 sq.

24 Quant au chêne en tant que représentant de la nature sauvage par rapport à l'espace humanisé, cf. Ph. BoRGEAUD, Recherches sur le dieu Pan, Rome, 1979 (Bibliotheca Helvetica Romana, 17), p. 30 sq.; J.-P. VERNANT, Manger au pays du soleil, in La cuisine, op.cit., p. 243 et n. 5. 
terre ${ }^{25}$, ce qui explique l'attachement inébranlable des prêtres de Zeus, des Selloi, à la terre de Dodone.

C'est dans cette perspective qu'il faut envisager le rôle de ces prêtres qui se distinguent par leur étrange pratique de ne jamais se laver les pieds et de dormir par terre ${ }^{26}$. L'habitude des desservants de l'oracle

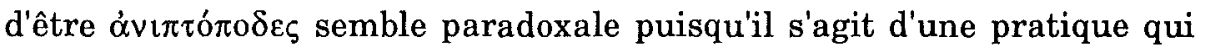
s'oppose à la propreté et la purification, éléments primordiaux dans le système religieux du monde hellénique ${ }^{27}$. Les qualités qui s'expriment au moyen des pieds nus, noirs ou boiteux témoignent d'un statut exceptionnel ${ }^{28}$ qui, dans le cas des Selloi ${ }^{29}$, est accentué encore davantage

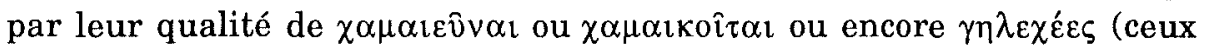
qui sont couchés sur le sol) qui se réfêre à leurs aptitudes divinatoires autant qu'à leur lien profond à la terre ${ }^{30}$.

25 Sur le rapport entre le chêne et l'autochtonie, cf. Y. VADÉ, Sur la maternité du chêne et de la pierre, in RHR, 191 (1977), p. 3-41.

26 Les pratiques étranges des Selloi qui couchaient sur le sol et ne se lavaient jamais les pieds (supra n. 2-3) ont été interprétées, soit comme étant les vestiges d'un état précivilisé (N.G.L. Hammond, Epirus. The Geography, the Ancient Remains, the History and the Topography of Epirus and Adjacent Areas, Oxford, 1967, p. 372; PöTsCHER, art. cit., p. 143), soit comme des pratiques liées à un dieu d'origine terrestre (CoOK, art. cit., in CJ, 17 [1903], p. 180; A. LESKY, Hellos-Hellotis, in WS, 46 [1927-1928], p. 48-68 et surtout p. 51-52; SAKELlaRIOU, op. cit., p. 105 et n. 5). Sur la même discussion, cf. aussi PARKE, op. cit., p. 1 sq.; Tsouvara-Souli, op. cit., n. 486, 487.

27 Cf. L. Moulinier, Le pur et l'impur dans la pensée des Grecs, Paris, 1952, p. 25 sq., 102 sq., 131 sq.; R.C.T. PARKER, Miasma. Pollution and Purification in Early Greek Religion, Oxford, 1983, p. 27 sq.

28 On se réfere ici au cas d'GEdipe (cf. J.-P. VERNANT, Le tyran boiteux : d'Édipe à Périandre, in Mythe et tragédie, II, Paris, 1986, p. 46 sq.) et de Mélampous, l'homme aux pieds noirs dont le lien avec la mantique, les oiseaux et le chêne est manifeste (APOLLOD., I, 9, 11).

29 Cf. aussi EVANs, art. cit., p. 107 sq. et E. IRWIN, Colour Terms in Greek Poetry, Toronto, 1974, p. $123 \mathrm{sq}$. - Les pieds nus fonctionnent donc, comme d'ailleurs les pieds noirs ou boiteux, sur plusieurs plans, à savoir attachement à la terre, autochtonie (sur la double provenance du mot Selloi, cf. Tsouvara-Souli, op. cit., n. 487), et divination, communication liées au savoir.

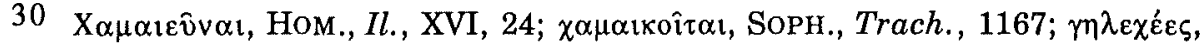
Callim., Hymne à Délos, 286. Déméter était honorée du titre de $\chi \alpha \mu v ́ v \eta$ à Olympie (PAus., VI, 20, 9 et 21, 1-2). 
De l'autre côté, les prêtresses 31 incontestablement présentes à l'oracle de Dodone se limitent à un rôle plutôt complémentaire avec une multitude de tâches. Ces femmes, connues sous le nom de Пé $\lambda \varepsilon \imath \alpha$ ou

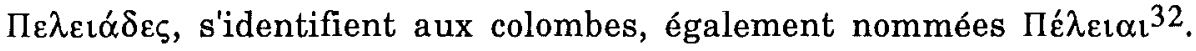
Elles se présentent tantôt comme de jeunes femmes à l'âge de la fécondité (comme les $\Pi \varepsilon \lambda \varepsilon \chi_{\alpha} \alpha \delta \varepsilon \varepsilon^{33}$ tantôt comme des femmes âgées ${ }^{34}$, destinées à accomplir uniquement leurs devoirs rituels. Cette ambivalence qui marque les prêtresses de l'oracle, femmes et colombes ou même femmes vieilles et jeunes à la fois, devient plus intelligible par

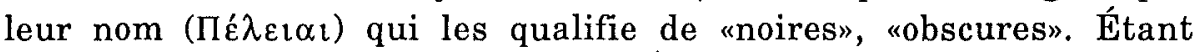
noires, ces femmes originaires d'Égypte retrouvent l'ambiance naturelle qui leur convient dans cette région d'Épire, qui s'appelait $\Sigma \kappa o \tau o v \sigma \sigma \sigma \alpha^{35}$, où habitaient les Dryopes, descendants de Mélaneus ou de Mé $\lambda \alpha \varsigma^{36}$. Elles s'intègrent aisément à l'atmosphère d'obscurité qui $\mathrm{y}$ règne.

Cette image se précise davantage si l'on se réfêre à l'exemple de l'Arcadie et aux rites analogues qui y avaient lieu et où le chêne, avec la présence simultanée de la colombe, s'attache au rituel de déesses noires. Et c'est là aussi qu'on peut déceler l'existence, au-delà de la mantique, d'une pratique sacrificielle qui marque l'étape transitoire de la vie sauvage à la vie civilisée ${ }^{37}$. Il faut cependant spécifier que, dans le cas de Dodone, cette pratique de la cuisine du sacrifice n'est pas aussi nette puisque l'intérêt primordial a toujours été centré sur la mantique. Sans indication précise à propos de la cuisine du sacrifice sur le territoire de Dodone, on peut tout de même affirmer que cette étape de transition est marquée par l'usage de chaudrons.

31 HÉRODOTE (II, 55) est le premier à nous présenter ces prêtresses dont l'aînée s'appelait Proméneia, la deuxième Timarété et la plus jeune Nicandra. Sur les qualifications multiples de ces femmes, cf. BoDSON, op. cit., p. 112, n. 152-154.

32 SopH., Trach., 172. Sur la discussion, cf. Bonson, op. cit., p. 106 sq.

33 À propos des Péleiades qui font communiquer les espaces humain et divin, cf. J.-P. VERNANT, La mètis orphique et la seiche de Thétis, in M. DETIENNE J.-P. Vernant, Les ruses de l'intelligence, Paris, 1974, p. 146 sq.; Ballabriga, op. cit., p. 98 sq.

34 Cf. STRAB., VII, fr. 1-2; Schol. SOPH., Trach., 172; HÉSYCH., s.v. Пé BODSON, op. cit., p. 106, n. 135.

35 Cf. SAKELLARIOU, op. cit., p. 161.

36 Cf. SAKELlariou, op. cit., p. 265 et n. 8.

37 Cf. L. BRUIT, Pausanias à Phigalie : Sacrifices non-sanglants et discours idéologique, in Métis, 1 (1986), p. 71-96. 
La découverte des chaudrons dans le site sacré de Dodone ${ }^{38}$ a ouvert une nouvelle perspective d'interprétation du fonctionnement de l'oracle. Il est évident que la fonction des chaudrons ne peut pas se borner à l'élément acoustique si souvent signalé par les scholiastes et les interprètes de l'oracle. Il s'agit des ustensiles employés par les sociétés qui utilisaient le feu et par conséquent le sacrifice et la nourriture cuisinée ${ }^{39}$. Bien que la majorité des récits relatifs à Dodone ne fassent pas mention de tels chaudrons, ils spécifient que les prêtresses étaient jetées dans l'eau bouillante ${ }^{40}$. Il s'agit d'une pratique qui faisait partie des rites relatifs au rajeunissement et à l'immortalisation ${ }^{41}$, c'est-à-dire de ces pratiques qui caractérisent l'effort de l'homme pour dépasser sa nature humaine et mortelle afin d'accéder au monde des immortels. Ainsi l'usage des chaudrons dans le cadre de l'oracle est polyvalent: liés au feu, ils sont de l'ordre de la culture et, en tant qu'agents de l'immortalisation et de la divination, ils réalisent la jonction entre l'humain et le divin ${ }^{42}$.

38 La tradition nous rapporte que les chaudrons étaient rangés en cercle autour du chêne et qu'ils prophétisaient grâce au son qui se répercutait d'un chaudron

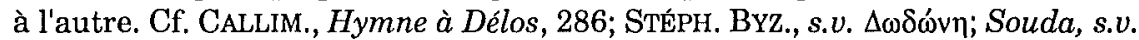

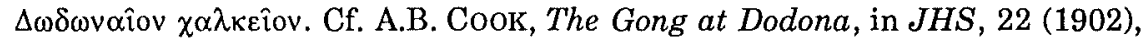

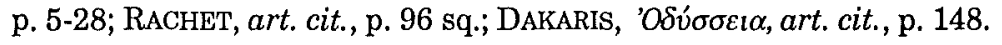

39 Sur le lien des chaudrons de bronze avec le monde masculin et l'alimentation carnée, cf. M. DETIENNE, Violentes eugénies, in La cuisine, op. cit., p. 190; A.-E. LAURENS, L'enfant entre l'épée et le chaudron : contribution à une lecture iconographique, in $D H A, 10$ (1984), p. 203-251 et surtout p. 228 sq.

40 Selon ÉPHORE (70 F 119, 4 J.), les Béotiens ont jeté la prophétesse dans le feu. De même selon EURIPIDE (Érechthée, fr. $368 \mathrm{Nauck}^{2}$ ), la prophétesse a été jetée par les Thébains dans l'eau bouillante. Cf. CooK, Zeus, II, p. 214, n. 3, p. 215, n. 1; PARKE, op. cit., p. 71 sq.; BODSON, op. cit., p. 112, n. 157. - COOK (art. cit., in CQ, 17 [1905], p. 190) a proposé aussi l'hypothèse de l'existence d'un xoanon de la déesse qui aurait été brûlé par le feu de Dodone, feu qui se manifeste à travers les chaudrons mais aussi à travers le chêne, la hache et la foudre (CoOK, Zeus, II, p. 676). Cette hypothèse a l'avantage de renvoyer à la fête béotienne des Daidala, cérémonie à désignation oraculaire. Sur les Daidala, cf. F. FronTisIDucroux, Dédale. Mythologie de l'artisan en Grèce ancienne, Paris, 1975, p. 193 sq.; A. SCHACHTER, Cults of Boiotia, London, 1981 (BICS, 38, 1), p. 245 sq. et bibliographie n. 3 .

41 On se réfere aux cas de Déméter, Médée, Thétis, etc. qui sont renommées pour leurs rajeunissements.

42 Cf. M. Detienne, Dionysos mis à mort, Paris, 1977, p. 180 sq. 
L'image que l'on se fait de l'oracle peut être complétée par la présence de la déesse Dioné, fille de l'Océan, qui accompagne Zeus ${ }^{43}$. Elle se présente comme parèdre de Zeus et elle est la déesse de la fécondité, la maîtresse des eaux et de la vie, d'où les qualifications de Zeus Nóiöo et de Dioné Naía qui impliquent qu'ils habitent l'oracle et qu'ils aient un rapport étroit avec l'eau ${ }^{44}$. L'association de Dioné et de Zeus dans le domaine de la fécondité autant que dans le domaine oraculaire, exprimée entre autres par le biais de sa communication étroite avec l'élément liquide ${ }^{45}$, annule toute possibilité d'une antériorité quelconque de la déesse par rapport au dieu de l'oracle ${ }^{46}$.

On peut donc dégager une image cohérente de l'oracle qui fonctionne à trois niveaux : dieux, oiseaux et homme-nature dont l'élément médian, soit les oiseaux, assure la communication entre les deux autres niveaux. De l'autre côté, on constate un échelonnement des problèmes divers de la condition humaine tels que l'alimentation et la communication polarisées autour du savoir de la vérité oraculaire. Aussi faut-il spécifier que la fonction de l'oracle révèle un système d'oppositions ou d'analogies au niveau religieux et social. On pourrait même discerner des couples d'opposition tels que Zeus et Dioné, $\Sigma \varepsilon \lambda \lambda$ ol et $\Pi \varepsilon \hat{\varepsilon} \lambda \varepsilon \imath \alpha$, aigle et colombe, ou encore l'opposition générale du mouvement du monde céleste à la stabilité du monde terrestre. Cette articulation symbolique de l'oracle devient plus claire si l'on tient compte des analogies telles que

43 À propos de Dioné, cf. BoDSON, op. cit., p. 114 sq.; Tsouvara-SoULI, op. cit., p. 68

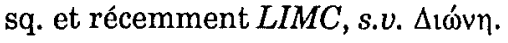

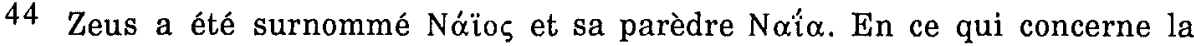
signification des verbes voí $\omega$ (habiter) et voí $\omega$ (couler), cf. PöTSCHER, art. cit., p. 10 sq. et DaKaris, Tò içóv, art. cit., p. 142 sq. - Ce qui demeure obscur, c'est le rituel de la fête des Nói $\alpha$, fête probablement de type initiatique, où les vainqueurs recevaient des trépieds et des couronnes de chêne. Cf. P. CABANES, L'Épire de la mort de Pyrrhos à la conquête romaine (272-167), Paris, 1976,

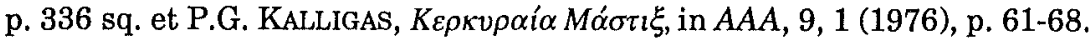

45 Dans la région d'Épire, l'eau de la vie et de la mort, l'eau de la fécondité et du savoir, aspects principaux de l'eau primordiale (cf. RUDHARDT, op. cit.) est figurée par le fleuve Achéron et la source qui coule près du sanctuaire (PLINE, N.H., II, 103 [228]).

46 La présence de Dioné, qui a donné naissance aux théories qui privilégient les puissances féminines par rapport aux puissances masculines de l'oracle (supra n. 8) ne peut être dissociée de Zeus dont les mariages avec des déesses du savoir affirment sa souveraineté et son pouvoir dans le domaine de la communication. Cf. M. DETIENNE - J.-P. VeRnANT, L'union avec Metis et la royaute du ciel, in Les ruses, op. cit., p. 104 sq.; A. IRIARTE, Savoir et pouvoir de Zeus, in Itaca, 2 (1986), p. 9-24. 
celle de la couleur entre les colombes et les femmes, le rapport commun à la terre des colombes, des Selloi et du chêne, le caractère sauvage du chêne et des oiseaux (glands, nectar-ambroisie) et enfin la dimension civilisatrice et divinatoire à la fois du feu et de l'eau.

Ainsi, nous constatons l'existence d'un système cohérent où s'expriment les plus grands problèmes touchant à l'organisation de la vie humaine, c'est-à-dire ceux de l'alimentation et de la communication.

L'intégration de tous ces problèmes dans le domaine de l'oracle manifeste non seulement l'établissement définitif de l'ordre cosmique qui sépare les immortels des mortels, mais aussi l'effort incessant de ces derniers pour participer au monde divin au travers du savoir que l'oracle révèle.

Université de Ioannina

Ariadni GARTZIOU-TATTI

Institut de Philologie classique GR - 45332 IOANNINA 